\title{
STATISTICAL MECHANICS OF THE SELF-GRAVITATING GAS: THERMODYNAMIC LIMIT, UNSTABILITIES AND PHASE DIAGRAMS
}

\author{
H. J. de Vega ${ }^{(b, a) 1}$ N. G. Sanchez ${ }^{(a) 2}$ \\ (a) Observatoire de Paris, LERMA, Laboratoire Associé au CNRS \\ UMR 8112, 61, Avenue de l'Observatoire, 75014 Paris, France. \\ (b) LPTHE, Laboratoire Associé au CNRS UMR 7589, \\ Université Pierre et Marie Curie (Paris VI) et Denis Diderot (Paris VII), \\ Tour 24, 5 ème. étage, 4, Place Jussieu, 75252 Paris, Cedex 05, France.
}

\begin{abstract}
We show that the self-gravitating gas at thermal equilibrium has an infinite volume limit in the three ensembles (GCE, CE, MCE) when $(N, V) \rightarrow \infty$, keeping $N / V^{1 / 3}$ fixed, that is, with $\eta \equiv \frac{G m^{2} N}{V^{1 / 3} T}$ fixed. We develop Monte Carlo simulations, analytic mean field methods (MF) and low density expansions. We compute the equation of state and find it to be locally $p(\vec{r})=T \rho_{V}(\vec{r})$, that is a local ideal gas equation of state. The system is in a gaseous phase for $\eta<\eta_{T}=1.51024 \ldots$ and collapses into a very dense object for $\eta>\eta_{T}$ in the $\mathrm{CE}$ with the pressure becoming large and negative. The isothermal compressibility diverges at $\eta=\eta_{T}$. We compute the fluctuations around mean field for the three ensembles. We show that the particle distribution can be described by a Haussdorf dimension $1<D<3$.

Nous montrons que la limite de volume infini existe pour le gaz autogravitant à l'equilibre thermique dans les trois ensembles (EGC,EC,EMC) quand $(N, V) \rightarrow \infty$, avec $N / V^{1 / 3}$ fixe, c'est à dire $\eta \equiv \frac{G m^{2} N}{V^{1 / 3} T}$ fixe. Nous utilisons les simulations Monte Carlo, la méthode du champ moyen et les developpements à basse densité. Nous calculons l'équation d'état et nous trouvons qu'elle est localement $p(\vec{r})=T \rho_{V}(\vec{r})$, c'est dire, l'quation d'un gaz parfait local. Le system est dans une phase gazeuse pour $\eta<\eta_{T}=1.51024 \ldots$ et s'effondre dans un objet très dense pour $\eta>\eta_{T}$ dans l'ensemble canonique avec une pression grande et négative. La compressibilité isothermique diverge à $\eta=\eta_{T}$. Nous calculons les fluctuations autour du champ moyen pour les trois ensembles. Nous montrons que la distribution des particules est décrite par une dimension de Haussdorf $1<D<3$.

\footnotetext{
${ }^{1}$ devega@lpthe.jussieu.fr

${ }^{2}$ Norma.Sanchez@obspm.fr
} 
Physical systems at thermal equilibrium are usually homogeneous. This is the case for gases with short range intermolecular forces (and in absence of external fields). In such cases the entropy is maximum when the system homogenizes.

When long range interactions as the gravitational force are present, even the ground state is inhomogeneous. In this case, each element of the substance is acted on by very strong forces due to distant particles in the gas. Hence, regions near to and far from the boundary of the volume occupied by the gas will be in very different conditions, and, as a result, the homogeneity of the gas is destroyed [1]. The state of maximal entropy for gravitational systems is inhomogeneous. This basic inhomogeneity suggested us that fractal structures can arise in a self-interacting gravitational gas [2, 3, 4, 6, 5].

The inhomogeneous character of the ground state for gravitational systems explains why the universe is not going towards a 'thermal death'. A 'thermal death' would mean that the universe evolves towards more and more homogeneity. This can only happen if the entropy is maximal for an homogeneous state. Instead, it is the opposite what happens, structures are formed in the universe through the action of the gravitational forces as time evolves.

Usual theorems in statistical mechanics break down for inhomogeneous ground states. For example, the specific heat may be negative in the microcanonical ensemble (not in the canonical ensemble where it is always positive) [1].

As is known, the thermodynamic limit for self-gravitating systems does not exist in its usual form $(N \rightarrow \infty, V \rightarrow \infty, N / V=$ fixed $)$. The system collapses into a very dense phase which is determined by the short distance (non-gravitational) forces between the particles. However, the thermodynamic functions exist in the dilute limit [3, 4, 6]

$$
N \rightarrow \infty, V \rightarrow \infty, \frac{N}{V^{1 / 3}}=\text { fixed }
$$

where $V$ stands for the volume of the box containing the gas. In such a limit, the energy $E$, the free energy and the entropy turns to be extensive. That is, we find that they take the form of $N$ times a function of the intensive dimensionless variables:

$$
\eta=\frac{G m^{2} N}{L T} \quad \text { or } \quad \xi=\frac{E L}{G m^{2} N^{2}}
$$

where $\eta$ and $\xi$ are intensive variables. Namely, $\eta$ and $\xi$ stay finite when $N$ and $V \equiv L^{3}$ tend to infinite. The variable $\eta$ is appropriate for the canonical 
ensemble and $\xi$ for the microcanonical ensemble. Physical magnitudes as the specific heat, speed of sound, chemical potential and compressibility only depend on $\eta$ or $\xi$. The variables $\eta$ and $\xi$, as well as the ratio $N / L$, are therefore intensive magnitudes. The energy, the free energy, the Gibbs free energy and the entropy are of the form $N$ times a function of $\eta$. These functions of $\eta$ have a finite $N=\infty$ limit for fixed $\eta$ (once the ideal gas contributions are subtracted). Moreover, the dependence on $\eta$ in all these magnitudes express through a single universal function $f(\eta)$. The variable $\eta$ is the ratio of the characteristic gravitational energy $\frac{G m^{2} N}{L}$ and the kinetic energy $T$ of a particle in the gas. For $\eta=0$ the ideal gas is recovered.

In refs. 3, 4] and 6] we have thoroughly studied the statistical mechanics of the self-gravitating gas. That is, our starting point is the partition function for non-relativistic particles interacting through their gravitational attraction in thermal equilibrium. We study the self-gravitating gas in the three ensembles: microcanonical (MCE), canonical (CE) and grand canonical (GCE). We performed calculations by three methods:

- By expanding the partition function through direct calculation in powers of $1 / \xi$ and $\eta$ for the MCE and CE, respectively. These expressions apply in the dilute regime $(\xi \gg 1, \eta \ll 1)$ and become identical for both ensembles for $N \rightarrow \infty$. At $\eta=0=1 / \xi$ we recover the ideal gas behaviour.

- By performing Monte Carlo simulations both in the MCE and in the $\mathrm{CE}$. We found in this way that the self-gravitating gas collapses at a critical point which depends on the ensemble considered. As shown in fig. 1 the collapse occurs first in the canonical ensemble (point T). The microcanonical ensemble exhibits a larger region of stability that ends at the point MC (fig. 1). Notice that the physical magnitudes are identical in the common region of validity of both ensembles within the statistical error. Beyond the critical point $\mathrm{T}$ the system becomes suddenly extremely compact with a large negative pressure in the CE. Beyond the point $\mathrm{MC}$ in the $\mathrm{MCE}$ the pressure and the temperature increase suddenly and the gas collapses. The phase transitions at $\mathrm{T}$ and at $\mathrm{MC}$ are of zeroth order since the Gibbs free energy has discontinuities in both cases.

- By using the mean field approach we evaluate the partition function for large $N$. We do this computation in the grand canonical, canonical 


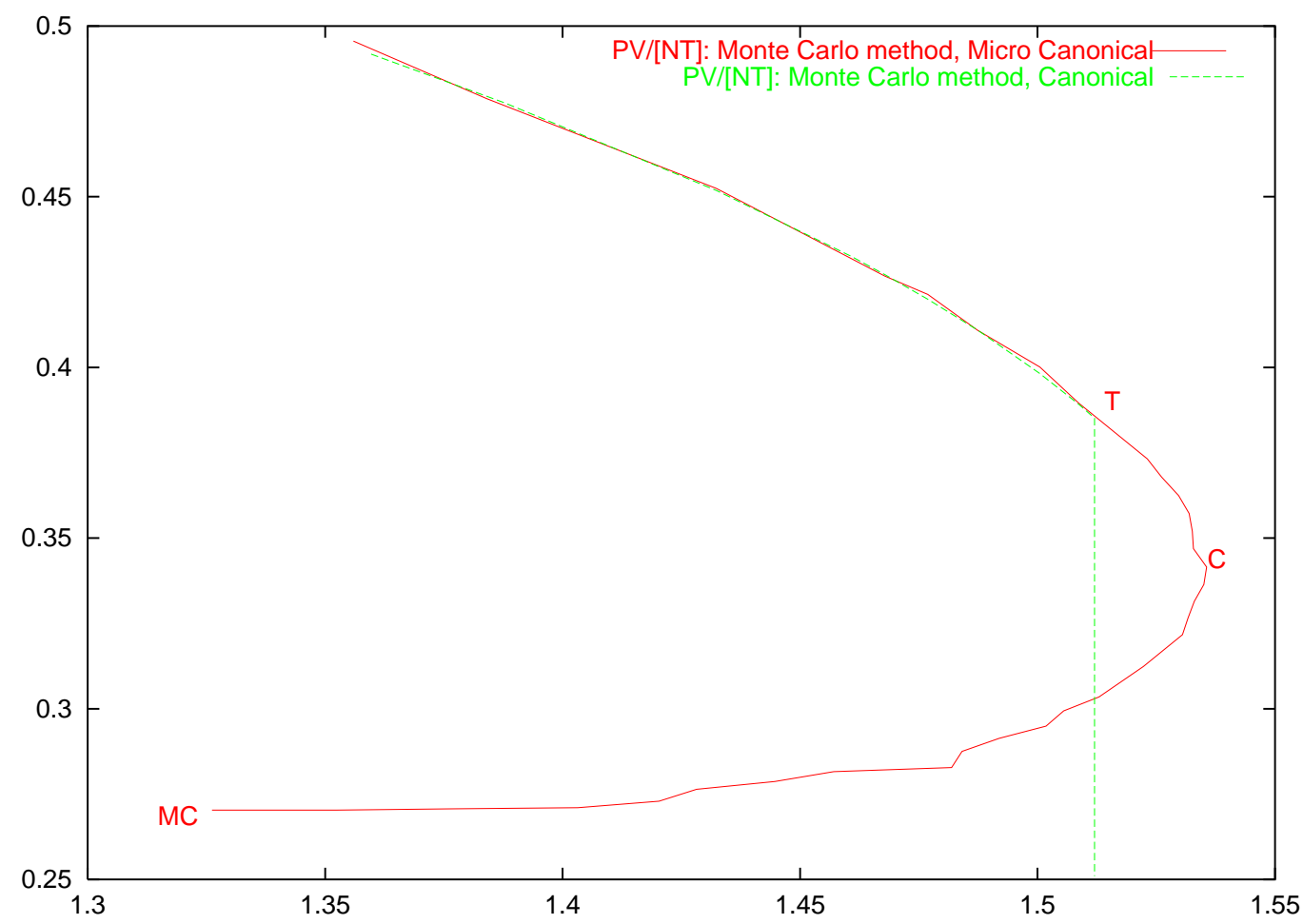

Figure 1: $f(\eta)=P V /[N T]$ as a function of $\eta$ by Monte Carlo simulations for the microcanonical and canonical ensembles $(N=2000)$. Both curves coincide within the statistical error till the point $\mathrm{T}$. 
and microcanonical ensembles. In the three cases, the partition function is expressed as a functional integral over a statistical weight which depends on the (continuous) particle density. These statistical weights are of the form of the exponential of an 'effective action' proportional to $N$. Therefore, the $N \rightarrow \infty$ limit follows by the saddle point method. The saddle point is a space dependent mean field showing the inhomogeneous character of the ground state. Corrections to the mean field are of the order $1 / N$ and can be safely ignored for $N \gg 1$ except near the critical points. These mean field results turned out to be in excellent agreement with the Monte Carlo results and with the low density expansion.

We calculate the saddle point (mean field) for spherical symmetry and we obtain from it the various physical magnitudes (pressure, energy, entropy, free energy, specific heats, compressibilities, speed of sound and particle density). Furthermore, we computed in ref. 4 the determinants of small fluctuations around the saddle point solution for spherical symmetry for the three statistical ensembles.

When any small fluctuation around the saddle point decreases the statistical weight in the functional integral, the saddle point dominates the functional integral and the mean field approach can be valid. In that case, the determinant of small fluctuations is positive. A negative determinant of small fluctuations indicates that some fluctuations around the saddle point are increasing the statistical weight in the functional integral and hence the saddle point does not dominate the partition function. The mean field approach cannot be used when the determinant of small fluctuations is negative. We find analytically in the CE that the determinant of small fluctuations vanishes at the point $\eta_{C}=1.561764$ and becames negative at $\eta>\eta_{C}$ [3. (The point $\eta_{C}$ is indicated $C$ in Figure 1). We find that the CE specific heat $c_{V}$ at the point $C$ diverges as [3]:

$$
c_{V} \stackrel{\eta \uparrow \eta_{C}}{=} \pm 0.63572 \ldots\left(\eta_{C}-\eta\right)^{-1 / 2}-0.19924 \ldots+O\left(\sqrt{\eta_{C}-\eta}\right)
$$

The $(+)$ sign refers to the positive (first) branch, and the (-) sign to the negative (second) branch (between the points $C$ and $M C$ ).

However, it must be noticed that the instability point is located at $\eta=\eta_{T}<\eta_{C}$, as shown by both, mean field and Monte Carlo computations. (The point $\eta_{T}$ is indicated $T$ in Figure 1 ). The onset of instability in the 
canonical ensemble coincides with the point where the isothermal compressibility diverges. The isothermal compressibility $\kappa_{T}$ is positive from $\eta=0$ till $\eta=\eta_{T}=1.51024 \ldots$. At this point $\kappa_{T}$ as well as the specific heat at constant pressure $c_{P}$ diverge and change their signs [3. Moreover, at this point the speed of sound at the center of the sphere becomes imaginary 4 . Therefore, small density fluctuations will grow exponentially in time instead of exhibiting oscillatory propagation. Such a behaviour leads to the collapse of the gas into a extremely compact object. Monte-Carlo simulations confirm the presence of this instability at $\eta_{T}=1.510 \ldots$ in the canonical ensemble and the formation of the collapsed object [3].

The collapse in the Grand Canonical ensemble (GCE) occurs for a smaller value of $\eta=\eta_{G C}=0.49465 \ldots$, while in the microcanonical ensemble, the collapse arrives later, in the second sheet, at $\eta=\eta_{M C}=1.25984 \ldots$.

We find that the Monte Carlo simulations for self-gravitating gas in the $\mathrm{CE}$ and the MCE confirm the stability results obtained from mean field.

The saddle point solution is identical for the three statistical ensembles. This is not the case for the fluctuations around it. The presence of constraints in the $\mathrm{CE}$ (on the number of particles) and in the MCE (on the energy and the number of particles) changes the functional integral over the quadratic fluctuations with respect to the GCE.

The saddle point of the partition function turns out to coincide with the hydrostatic treatment of the self-gravitating gas [7] (which is usually known as the 'isothermal sphere' in the spherically symmetric case).

We find that the Monte Carlo simulations (describing thermal equilibrium) are much more efficient than the $N$-body simulations integrating Newton's equations of motion. (Indeed, the integration of Newton's equations provides much more detailed information than the one needed in thermal equilibrium investigations). Actually, a few hundreds of particles are enough to get quite accurate results in the Monte Carlo simulations (except near the collapse points). Moreover, the Monte Carlo results turns to be in excellent agreement with the mean field calculations up to very small corrections of the order $(1 / N)$. Our Monte Carlo simulations are performed in a cubic geometry. The equilibrium configurations obtained in this manner can thus be called the 'isothermal cube'.

In summary, the picture we get from our calculations using these three methods show that the self-gravitating gas behaves as a perfect gas for $\eta \rightarrow 0,1 / \xi \rightarrow 0$. When $\eta$ and $1 / \xi$ grow, the gas becomes denser till it suddenly condenses into a high density object at a critical point GC, C or 


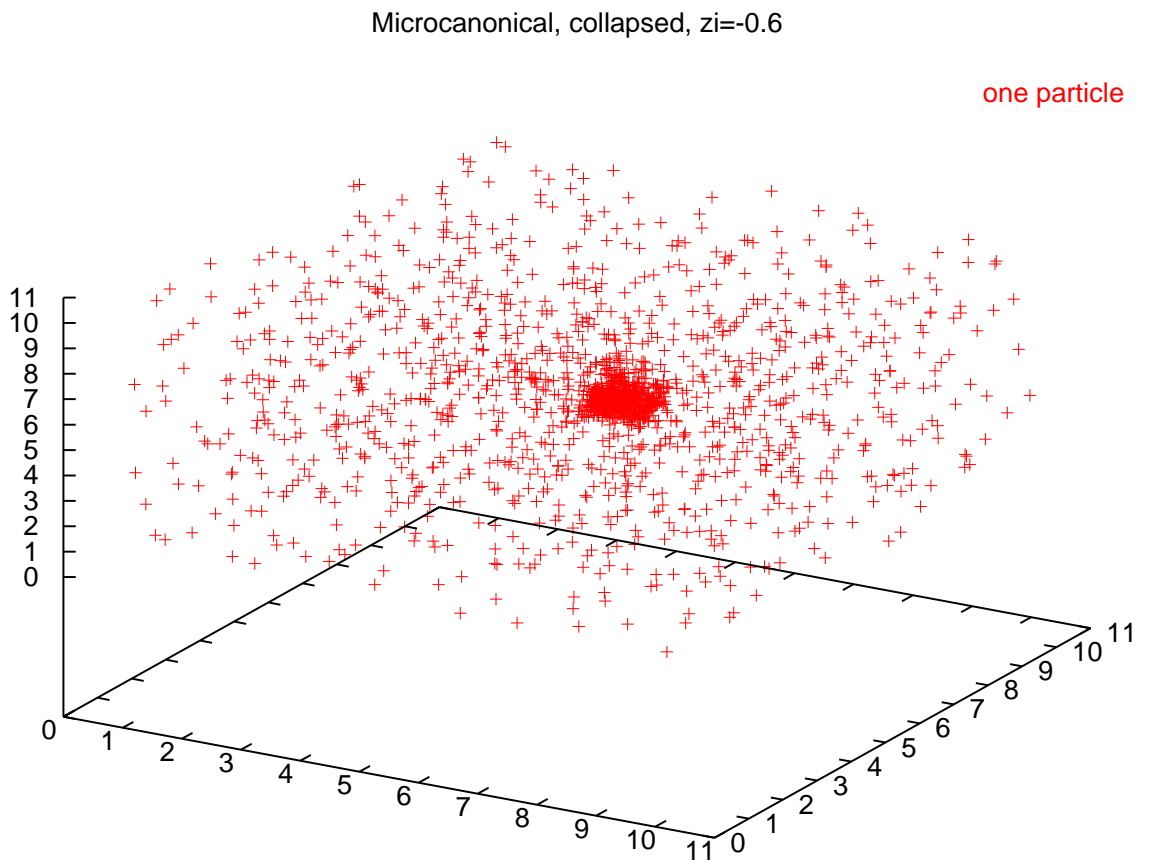

Figure 2: Average particle distribution in the collapsed phase from Monte Carlo simulations with 2000 particles in the microcanonical ensemble for $\xi=-0.6, \eta=0.43, p V /[N T]=0.414$.

MC depending upon the statistical ensemble chosen.

$\eta$ is related with the Jeans' length $d_{J}$ of the gas through $\eta=3\left(L / d_{J}\right)^{2}$. Hence, when $\eta$ goes beyond $\eta_{T}$, the length of the system becomes larger than $d_{J} / \sqrt{\eta_{T} / 3}$. The collapse at $\mathrm{T}$ in the $\mathrm{CE}$ is therefore a manifestation of the Jeans' instability.

In the MCE, the determinant of fluctuations vanishes at the point MC. The physical states beyond MC are collapsed configurations as shown by the Monte Carlo simulations (see fig. 2). Actually, the gas collapses in the Monte Carlo simulations slightly before the mean field prediction for the point MC. The phase transition at the microcanonical critical point $\mathrm{MC}$ is the so called gravothermal catastrophe [8].

The gravitational interaction being attractive without lower bound, a short distance cut-off $(A)$ must be introduced in order to give a meaning to 
the partition function. We take the gravitational force between particles as $-G m^{2} / r^{2}$ for $r>A$ and zero for $r<A$ where $r$ is the distance between the two particles. We show that the cut-off effects are negligible in the $N=\infty$ limit. That is, once we set $N=\infty$ with fixed $\eta$, all physical quantities are finite in the zero cut-off limit $(A=0)$. The cut-off effects are of the order $A^{2} / L^{2}$ and can be safely ignored.

In ref. [3] we expressed all global physical quantities in terms of a single function $f(\eta)$. Besides computing numerically $f(\eta)$ in the mean field approach, we showed that this function obeys a first order non-linear differential equation of first Abel's type. We obtained analytic results about $f(\eta)$ from the Abel's equation. $f(\eta)$ exhibits a square-root cut at $\eta_{C}$, the critical point in the $\mathrm{CE}$. The first Riemann sheet is realized both in the $\mathrm{CE}$ and the MCE, whereas the second Riemann sheet (where $c_{V}<0$ ) is only realized in the MCE. $f(\eta)$ has infinitely many branches in the $\eta$ plane but only the first two branches are physically realized. Beyond MC the states described by the mean field saddle point are unstable.

We plot and analyze the equation of state, the energy, the entropy, the free energy, $c_{V}$ and the isothermal compressibility (figs. 9-13 in ref. [3]). Most of these physical magnitudes were not previously computed in the literature as functions of $\eta$.

We find analytically the behaviour of $f(\eta)$ near the point $\eta_{C}$ in mean field,

$f(\eta) \stackrel{\eta \uparrow \eta_{C}}{=} \frac{1}{3}+0.27137 \ldots \sqrt{\eta_{C}-\eta}+0.27763 \ldots\left(\eta_{C}-\eta\right)+O\left[\left(\eta_{C}-\eta\right)^{3 / 2}\right]$.

This exhibits a square root branch point singularity at $\eta_{C}$. This shows that the specific heat at constant volume diverges at $\eta_{C}$ as $\left(\eta_{C}-\eta\right)^{-1 / 2}$ for $\eta \uparrow \eta_{C}$. However, it must be noticed, that the specific heat at constant pressure and the isothermal compressibility? both diverge at the point $\eta_{T}$ as $\left(\eta_{T}-\eta\right)^{-1}$. These mean field results apply for $\left|\eta-\eta_{C}\right| \ll 1 \ll N\left|\eta-\eta_{C}\right|$. Fluctuations around mean field can be neglected in such a regime.

The Monte Carlo calculations permit us to obtain $f(\eta)$ in the collapsed phase. Such result cannot be obtained in the mean field approach. The mean field approach only provides information as $f(\eta)$ in the dilute gas phase.

For the self-gravitating gas, we find that the Gibbs free energy $\Phi$ is not equal to $N$ times the chemical potential and that the thermodynamic potential $\Omega$ is not equal to $-P V$ as usual [1]. This is a consequence of the dilute thermodynamic limit $N \rightarrow \infty, L \rightarrow \infty, N / L=$ fixed. 
We computed the determinant of small fluctuations around the saddle point solution for spherical symmetry in all three statistical ensembles. In the spherically symmetric case, the determinant of small fluctuations is written as an infinite product over partial waves. The $\mathrm{S}$ and $\mathrm{P}$ wave determinants are written in closed form in terms of the saddle solution. The determinants for higher partial waves are computed numerically. All partial wave determinants are positive definite except for the S-wave [4. The reason why the fluctuations are different in the three ensembles is rather simple. The more contraints are imposed the smaller becomes the space of fluctuations. Therefore, in the grand canonical ensemble (GCE) the system is more free to fluctuate and the phase transition takes place earlier than in the microcanonical (MCE) and canonical ensembles (CE). For the same reason, the transition takes place earlier in the $\mathrm{CE}$ than in the MCE.

The conclusion being that the mean field correctly gives an excellent description of the thermodynamic limit except near the critical points (where the small fluctuations determinant vanishes); the mean field is valid for $N \mid \eta-$ $\eta_{\text {crit }} \mid \gg 1$. The vicinity of the critical point should be studied in a double scaling limit $N \rightarrow \infty, \eta \rightarrow \eta_{\text {crit }}$. Critical exponents are reported in ref. 3] for $\eta \rightarrow \eta_{C}$ using the mean field. These mean field results apply for $\left|\eta-\eta_{C}\right| \ll 1 \ll N\left|\eta-\eta_{C}\right|$ with $N \gg 1$. Fluctuations around mean field can be neglected in such a regime.

We computed local properties of the gas in 4. That is, the local energy density $\epsilon(r)$, local particle density, local pressure and the local speed of sound. Furthermore, we analyze the scaling behaviour of the particle distribution and its fractal (Haussdorf) dimension [4].

The particle distribution $\rho_{V}(\vec{q})$ proves to be inhomogeneous (except for $\eta \ll 1)$ and described by an universal function of $\eta$, the geometry and the ratio $\vec{r}=\vec{q} / R, R$ being the radial size. Both Monte Carlo simulations and the Mean Field approach show that the system is inhomogeneous forming a clump of size smaller than the box of volume $V$ [see fig. 2 here and figs. 3, 5 and 6 in [3]].

The particle density in the bulk behaves as $\rho_{V}(\vec{q}) \simeq r^{D-3}$. That is, the mass $M(R)$ enclosed on a region of size $R$ vary approximately as

$$
M(R) \simeq C R^{D} .
$$

$D$ slowly decreases from the value $D=3$ for the ideal gas $(\eta=0)$ till $D=0.98$ in the extreme limit of the MC point, $D$ takes the value 1.6 at 
$\eta_{C}$, [see Table 1]. This indicates the presence of a fractal distribution with Haussdorf dimension $D$.

Our study of the statistical mechanics of a self-gravitating system indicates that gravity provides a dynamical mechanism to produce fractal structures [2, 3, 4].

The average distance between particles monotonically decrease with $\eta$ in the first sheet. The mean field and Monte Carlo are very close in the gaseous phase whereas the Monte Carlo simulations exhibit a spectacular drop in the average particle distance at the clumping transition point $\mathrm{T}$. In the second sheet (only described by the MCE) the average particle distance increases with $\eta[4$.

We find that the local equation of state is given by

$$
p(\vec{r})=T \rho_{V}(\vec{r}) .
$$

We have derived the equation of state for the self-gravitating gas. It is locally the ideal gas equation, but the self-gravitating gas being inhomogeneous, the pressure at the surface of a given volume is not equal to the temperature times the average density of particles in the volume. In particular, for the whole volume: $P V /[N T]=f(\eta) \leq 1$ (the equality holds only for $\eta=0)$.

Notice that we have found the local ideal gas equation of state $p(\vec{r})=$ $T \rho_{V}(\vec{r})$ for purely gravitational interaction between particles. Therefore, equations of state different from this one, (as often assumed and used in the literature for the self-gravitating gas), necessarily imply the presence of additional non-gravitational forces.

The local energy density $\epsilon(r)$ turns out to be an increasing function of $r$ in the spherically symmetric case. The energy density is always positive on the surface, whereas it is positive at the center for $0 \leq \eta<\eta_{3}=1.07783 \ldots$, and negative beyond the point $\eta=\eta_{3}=1.07783 \ldots$

The local speed of sound $v_{s}^{2}(r)$ is computed in the mean field approach as a function of the position for spherical symmetry and long wavelengths. $v_{s}^{2}(r)$ diverges at $\eta=\eta_{T}=1.51024 \ldots$ in the first Riemann sheet. Just beyond this point $v_{s}^{2}(r)$ is large and negative in the bulk showing the strongly unstable behaviour of the gas for such range of values of $\eta$.

Moreover, we have shown the equivalence between the statistical mechanical treatment in the mean field approach and the hydrostatic description of the self-gravitating gas [7]. 
The success of the hydrodynamical description depends on the value of the mean free path $(l)$ compared with the relevant sizes in the system. $l$ must be $\ll 1$. We compute the ratio $l / a$ (Knudsen number), where $a$ is a length scale that stays fixed for $N \rightarrow \infty$ and show that $l / a \sim N^{-2}$. This result ensures the accuracy of the hydrodynamical description for large $N$.

Furthermore, we have computed in ref. 44 several physical magnitudes as functions of $\eta$ and $r$ which were not previously computed in the literature as the speed of sound, the energy density, the average distance between particles and we notice the presence of a Haussdorf dimension in the particle distribution.

The statistical mechanics of a selfgravitating gas formed by particles with different masses is thoroughly investigated in ref. [5] while the selfgravitating gas in the presence of the cosmological constant is thoroughly investigated in refs. 9]. In ref. [10 the Mayer expansion for the selfgravitating gas is investigated in connection with the stability of the gaseous phase.

\begin{tabular}{|l|l|l|}
\hline$\eta$ & $D$ & $C$ \\
\hline 0.06204 & 2.97 & 1.0 \\
\hline$\eta_{G C}=0.49465 \ldots$ & 2.75 & 1.03 \\
\hline 1.24070 & 2.22 & 1.1 \\
\hline$\eta_{C}=1.561764 \ldots$ & 1.60 & 1.07 \\
\hline$\eta_{M C}=1.25984 \ldots$ & 0.98 & 1.11 \\
\hline
\end{tabular}

TABLE 1. The Fractal Dimension $D$ and the proportionality coefficient $C$ as a function of $\eta$ from a fit to the mean field results according to $M(r) \simeq$ $C r^{D}$. The point $\eta_{M C}$ is on the second Riemann sheet.

\section{References}

[1] L. D. Landau, L. D., Lifchitz, E. M., 1996, Physique Statistique, 4ème édition, Mir-Ellipses

[2] de Vega, H. J., Sánchez, N., Combes, F., 1996, Nature, 383, 56, Phys. Rev. D54, 6008, 1998 Ap. J. 500, 8

[3] de Vega, H. J., Sánchez, N., 'Statistical Mechanics of the Self-Gravitating Gas and Fractal Structures. I', 2002, Nuclear Physics B 625, 409 
[4] de Vega, H. J., Sánchez, N., 'Statistical Mechanics of the Self-Gravitating Gas and Fractal Structures. II', 2002, Nuclear Physics B 625, 460

[5] de Vega, H. J., J. A. Siebert, J. A., 2002, Phys. Rev. E 66, 016112

[6] de Vega, H. J., Sánchez, N., astro-ph/0505561, 'Statistical Mechanics of the self-gravitating gas: thermodynamic limit, phase diagrams and fractal structures', astro-ph/0505561, 9th Course of the International School of Astrophysics 'Daniel Chalonge', Palermo, Italy, 7-18 September 2002, NATO ASI, p. 291-324 in the Proceedings, N. Sánchez and Yu. Parijskij editors, Kluwer, 2002.

[7] J. Binney and S. Tremaine, Galactic Dynamics, Princeton Univ. Press.

[8] Lynden-Bell, D. and Wood, R. , 1968, Mon. Not. R. astr. Soc. 138, 495

[9] de Vega, H. J., J. A. Siebert, J. A., 2005 Nucl. Phys. B 707, 529, B 726 [FS] 464

[10] de Vega, H. J., Sánchez, N., 2005, Nucl. Phys. B711, 604 